\title{
ТАРГЕТНОЕ СЕКВЕНИРОВАНИЕ У БОЛЬНЫХ С КЛИНИЧЕСКИ ДИАГНОСТИРОВАННЫМ НАСЛЕДСТВЕННЫМ НАРУШЕНИЕМ ЛИПИДНОГО ОБМЕНА И ОСТРЫМ КОРОНАРНЫМ СИНДРОМОМ
}

\author{
А. О. Аверкова ${ }^{\natural}$, В. А. Бражник ${ }^{1,2}$, Г. И. Спешилов ${ }^{3,4}$, \\ С. Н. Терещенко ${ }^{6}$, О. И. Боева ${ }^{7}$, Д. А. Затейщиков ${ }^{1,2}$ \\ ' Центральная государственная медицинская академия Управления делами Президента РФ, Москва \\ гГородская клиническая больница № 51, Москва \\ ${ }^{3}$ Институт проблем передачи информации имени А. А. Харкевича РАН, Москва \\ ${ }^{4}$ ООО «Ридсенс», Троицкий наноцентр ФИОП «Роснано», Москва \\ Казанский государственный медицинский университет, Казань \\ ${ }^{6}$ Национальный медицинский исследовательский центр кардиологии, Москва \\ Ставропольский государственный медицинский университет, Ставрополь
}

\begin{abstract}
Истинная распространенность генетических вариантов, стоящих за развитием семейной гиперхолестеринемии (СГХС) и характерных для каждой популяции, остается неизвестной. Целью работы было определение спектра патологических генетических вариантов у больных острым коронарным синдромом (ОКС) с клинически диагностированной СГХС с помощью таргетного секвенирования. Были отобраны 38 из 2081 пациентов двух многоцентровых наблюдательных исследований больных OKC (2004-2007; 2014-2016 гг.) возрастом $\leq 55$ лет (мужчины) и 560 лет (женщины) с СГХС, клинически диагностированной по критериям Голландской сети липидных клиник и критериям регистра Simon Broome. Молекулярно-генетическое исследование проводили с помощью таргетного секвенирования следующего поколения: сначала секвенировали 3 гена, ассоциированные с СГХС: $L D L R, A P O B, P C S K 9 ;$ при отсутствии значимых изменений панель расширяли. Из 38 пациентов у 24 (63,2\%) были выявлены генетические изменения, которые могли обусловить клинические проявления СГХС и раннюю манифестацию ишемической болезни сердца. Все пациенты являлись гетерозиготными носителями генетических вариантов. Выявлены варианты в трех основных генах (LDLR, APOB, PCSK9), связанных с СГХС, и редкие варианты в других генах системы липидного обмена (APOE, ABCA1, ABCG5, ABCG8, LPL, ANGPTL3, MTTP). Пять генетических вариантов описаны впервые: патогенный вариант p.Val273_Cys313del гена LDLR; вероятно патогенный вариант p.Arg160His гена APOE; варианты неуточненной клинической значимости p.Glu612Lys и c. *415G>A гена PCSK9; вариант p.Ala776Ser гена LDLR. Taким образом, использование клинических критериев позволяет выявить среди пациентов с ОКС и СГХС носителей мутаций не только «классических» генов, связанных с СГХС, но и редких, способных приводить К фенотипическим проявлениям СГХС.
\end{abstract}

Ключевые слова: семейная гиперхолестеринемия, острый коронарный синдром, генетическое обследование, нарушение липидного обмена, таргетное секвенирование

$\bowtie$ Для корреспонденции: Анастасия Олеговна Аверкова ул. Маршала Тимошенко, д. 19, стр. 1А, г. Москва, 121359; avek@mail.ru

Статья получена: 30.09.2018 Статья принята к печати: 05.11.2018 DOI: $10.24075 /$ vrgmu.2018.061

\section{TARGETED SEQUENCING IN PATIENTS WITH CLINICALLY DIAGNOSED HEREDITARY LIPID METABOLISM DISORDER AND ACUTE CORONARY SYNDROME}

Averkova $A O^{1} \otimes$, Brazhnik VA ${ }^{1,2}$, Speshilov Gl ${ }^{3,4}$, Rogozhina $A A^{1}$, Koroleva OS ${ }^{1}$, Zubova $\mathrm{EA}^{2}$, Galyavich $\mathrm{AS}^{5}$, Tereshenko $\mathrm{SN}^{6}$, Boyeva $\mathrm{Ol}^{7}$, Zateyshchikov DA ${ }^{1,2}$

Central State Medical Academy of the Department of Presidential Affairs of the Russian Federation, Moscow

${ }^{2}$ City Clinical Hospital No 51, Moscow

${ }^{3}$ Kharkevich Institute for Information Transmission Problems, RAS, Moscow

${ }^{4}$ ReadSense O००, Troitsk Center for Nanotechnologies of Rusnano Foundation for Nanotechnology Infrastructure and Educational Projects, Moscow

${ }^{5}$ Kazan State Medical University, Kazan

${ }^{6}$ National Medical Research Center for Cardiology, Moscow

7 Stavropol State Medical University, Stavropol

The actual prevalence of genetic variants causing familial hypercholesterolemia (FH) in every population remains unknown. The aim of this work was to determine the spectrum of pathogenic variants in patients with acute coronary syndrome (ACS) and clinically diagnosed FH using targeted sequencing. We selected 38 patients with ACS from the sample of 2,081 participants of two multicenter observational studies (2004-2007; 2014-2016) who had a clinical diagnosis of FH based on the Dutch Lipid Clinic Network score and Simon Broome criteria. The men and women included in the study were $\leq 55$ and $\leq 60$ years of age, respectively. Molecular genetic screening was done by targeted next-generation sequencing. We started by sequencing 3 genes associated with $\mathrm{FH}$, including $L D L R, A P O B$, and $P C S K 9$. If no relevant variants were detected, the panel was expanded. Of 38 patients, 24 (63.2\%) were shown to have mutations that could cause clinical manifestations of $\mathrm{FH}$ and premature coronary artery disease. All patients were heterozygous carriers. Mutations were detected in three "classic" genes $\angle D L R, A P O B$, and PCSK9 associated with $\mathrm{FH}$, as well as in other genes involved in lipid metabolism, such as APOE, ABCA1, ABCG5, ABCG8, LPL, ANGPTL3, and MTTP. Five variants detected in our study sample had not been described previously: the pathogenic p.Val273_Cys313del variant of the LDLR gene, the likely pathogenic p.Arg160His variant in the APOE gene, two variants of uncertain significance p.Glu612Lys and c. ${ }^{*} 415 \mathrm{G}>\mathrm{A}$ in the PCSK9 gene, and the mutant variant p.Ala776Ser in the LDLR gene. We conclude that the use of clinical diagnostic criteria in patients with ACS and FH enables identification of carriers of both "classic" mutations associated with $\mathrm{FH}$ and rare genetic variants that can be phenotypically expressed as $\mathrm{FH}$.

Keywords: familial hypercholesterolemia, acute coronary syndrome, genetic testing, lipid metabolism disorder, targeted sequencing

$\triangle$ Correspondence should be addressed: Anastasia O. Averkova

Marshala Timoshenko 19, b. 1A, Moscow, 121359; avek@mail.ru

Received: 30.09.2018 Accepted: 05.11.2018

DOI: 10.24075/brsmu.2018.061 
Среди наследственных нарушений липидного обмена семейная гиперхолестеринемия (СГХС) служит одной из наиболее известных причин ранней манифестации ишемической болезни сердца (ИБС) и ведет $k$ двадцатикратному повышению риска развития сердечнососудистых заболеваний [1]. Это самое распространенное моногенное нарушение липидного обмена с аутосомнодоминантным типом наследования, ведущее к повышению уровня холестерина липопротеидов низкой плотности (ЛНП). Большинство (60-80\%) больных гетерозиготной формой СГХС, определенной клинически, являются носителями мутаций в гене рецептора ЛНП (LDLR); в разных географических регионах преобладают различные мутации. В результате происходит уменьшение количества молекул рецептора ЛНП (ЛНПР) или снижение активности ЛНПР. Ген LDLR расположен в коротком плече 19 хромосомы и состоит из 18 экзонов, транскрипция и трансляция которых приводит к синтезу 5 доменов, формирующих на поверхности клетки ЛНПР [2]. Около 5-10\% больных гетерозиготной формой СГХС являются носителями мутаций гена $A P O B$, что проявляется фенотипически менее выраженным повышением уровня холестерина, чем в случае мутаций $L D L R$, и эти мутации более распространены у жителей центральной Европы, чем в других регионах [3]. Дефект белка апоВ100 на частицах ЛНП препятствует их связыванию с ЛНПР. Ген картирован на хромосоме 2р и включает 29 экзонов [2]. Мутации приобретения функции (gain-of-function, GOF) гена пропротеиновой конвертазы субтилизинкексинового типа 9 (PCSK9) представляют собой третью основную причину развития аутосомнодоминантной СГХС, обусловливая менее $1 \%$ случаев СГХС в большинстве исследованных популяций. Они приводят к ускоренной интернализации и разрушению ЛНПР и уменьшению количества молекул ЛНПР. Ген PCSK9 расположен в малом плече хромосомы 1р32 и содержит 12 экзонов и 11 интронов $[4,5]$. Очень редко причиной развития СГХС являются мутации в других генах, участвующих в обмене липидов [3]. Нередко пациенты оказываются носителями двух мутаций в генах, ассоциированных с СГХС. Однако у 20-40\% больных с клинически диагностированной СГХС причинную мутацию выявить не удается.

Целью работы было определение спектра патологических вариантов генов системы липидного обмена у больных острым коронарным синдромом (ОКС) с клинически диагностированной СГХС с помощью таргетного секвенирования.

\section{ПАЦИЕНТЫ И МЕТОДЫ}

В работе использованы данные больных, отобранных из 2081 участника двух многоцентровых (4 центра в трех городах - Москва, Казань, Ставрополь) наблюдательных исследований больных с ОКС, включение в которые проходило с 2004 по 2007 и с 2014 по 2016 г. Протокол исследования подробно описан ранее [6]. Проведение исследования одобрено решением заседания этического комитета ФГБУ «Учебно-научный медицинский центр» Управления делами Президента РФ (протокол № 14/14 от 20 октября 2014 г.

Для данной работы из первого периода набора отобрано 326 больных, из второго - 374. В течение первого периода набора критериями включения пациентов были: «раннее» развитие ОКС (в возрасте $\leq 55$ лет у мужчин и $\leq 60$ лет у женщин); развитие ОКС не ранее 10 дней до индексной госпитализации; наличие СГХС, диагностированной по критериям Голландской сети липидных клиник (КГСЛК) и регистра Simon Broome [7, 8]; наличие подписанного информированного согласия на участие в исследовании. Критерии включения во время второго периода: те же плюс необходимость проведения чрескожного коронарного вмешательства (ЧКВ) вне зависимости от итогового решения о его проведении у пациентов с ОКС.

СГХС диагностировали по критериям КГСЛК и регистра Simon Broome. По КГСЛК учитывается отягощенная наследственность, раннее развитие ИБС у самого больного, данные осмотра (сухожильные ксантомы, липоидная дуга роговицы), а также уровень ЛНП. При оценке данных, если больной набирает больше 8 баллов, речь идет об определенной СГХС, 6-8 баллов говорят о вероятной СГХС, 3-5 баллов свидетельствуют о возможной СГХС, а менее 3 баллов - диагноз СГХС маловероятен [7]. Согласно критериям регистра Simon Broome, выявление СГХС основывается на индивидуальной оценке уровня общего холестерина и ЛНП в зависимости от возраста больного и степени родства с тем, у кого этот уровень также повышен. В клинической практике, согласно данным критериям, возможна диагностика лишь возможной СГХС, так как диагноз определенной СГХС требует выявления причинной мутации [8].

Для проведения молекулярно-генетического исследования были отобраны 38 пациентов по следующему алгоритму. Первая часть исследования: все пациенты, набравшие $\geq 5$ баллов по КГСЛК, и все набравшие 4 балла по КГСЛК + пациенты с СГХС, диагностированной по регистру Simon Broome (всего 10 человек). Вторая часть исследования: все набравшие $\geq 5$ баллов по КГСЛК + пациенты С СГХС, диагностированной по регистру Simon Broome, и с отягощенной наследственностью (всего 24 человека). Из второй части исследования в анализ были также включены 4 пациента, которые в индексную госпитализацию не подходили под критерий наличия «раннего» ОКС, однако у которых был известен факт ранней манифестации ИБС ( $\geq 6$ баллов по КГСЛК и 5 баллов по КГСЛК + отягощенная наследственность).

Молекулярно-генетическое исследование проводили В генетической лаборатории ООО «Ридсенс» (Москва). Выделение ДНК выполняли посредством наборов для выделения геномной ДНК из крови на спин-колонках К-Сорб (Синтол; Россия). Синтез ДНК-библиотек из ДНК образцов для последующего секвенирования на секвенаторе нового поколения Illumina MiSeq (Illumina, США) производили с использованием набора NEB Next Ultra (NEB; CШA). Обогащение целевыми фрагментами генов (кодирующими областями) с использованием методики селективного захвата участков ДНК с помощью синтезированных зондов проводили с использованием таргетной синтетической панели обогащения NimbleGen (Roche; США). Проверку качества амплифицированной обогащенной ДНК-библиотеки выполняли провометодом капиллярного гель-электрофореза с использованием прибора BioAnalyzer 2100 (Roche; Швейцария). Контроль качества амплисицированной обогащенной ДНКбиблиотеки производили с использованием ДНКфллуориметра Qubit (Invitrogen; США) и капиллярного электрофореза BioAnalizer (Agilent; США). Далее проводили запуск секвенатора Illumina MiSeq в соответствии с рекомендациями производителя с использованием набора реактивов на 300 циклов (MiSeq 300 cycles v2). 
Сначала во всех исследуемых образцах проводили исследование трех генов, ассоциированных с СГХС: $L D L R$, APOB, PCSK9. Затем при отсутствии значимых генетических изменений панель расширяли с исследованием следующих генов, ассоциированных с обменом липидов: $A P O A 1$, APOA5, APOC2, APOE, APOC3, ABCA1, ABCG1, ABCG5, ABCG8, ANGPTL3, CEL, CH25H, CPT2, CYP2D6, CYP3A4, CYP3A5, GPD1, GPIHBP1, INSIG2, LCAT, LDLRAP1, LIPA, LMF1, LPA, LPL, MTTP, NPC1L1, PNPLA2, PPARA, SAR1B.

Проверка качества панели зондов включала следующие стадии для каждого из образцов: картирование прочтений секвенирования на референсный геном человека (сборка GRCh37.p13 hg19), расчет эффективности покрытия на основе данных о целевых фррагментах (для анализа брали нуклеотиды с минимальным покрытием 20х), используемых для синтеза зондов, поиск целевых мутаций в рамках панели обогащения, аннотацию найденных мутаций с помощью баз данных (HGMD, COSMIC, ClinVar, 1000 GenomesProject, dbSNP, ExAC), оценку консервативности замен с помощью методов моделирования (POLYPHEN SIFT, MUTATION TASTER, FATHMM, CADD, DANN, EIGEN). Аннотацию вариаций проводили в соответствии с рекомендациями АCMG [9].

\section{РЕЗУЛЬТАТЫ ИССЛЕДОВАНИЯ}

Все пациенты с выявленными изменениями гетерозиготны. По результатам проведенного обследования, мутации в трех «классических» генах, ответственных за развитие СГХС, были выявлены у 11 пациентов.

Среди них три патогенных и вероятно патогенных варианта в гене $L D L R$, а также один с неуточненной клинической значимостью. Патогенная мутация p.Val273 Cys313del ранее не описана и приводит к делеции внутри рамки считывания в области 6 экзона, в результате чего в ЛНПР отсутствует домен класса A7 в N-концевом участке [10]. Данная мутация относится к третьему классу мутаций гена $L D L R$, ведущих к синтезу рецептора с нарушением его связывания с ЛНП. Мутация g.11218068_11218190del с той же аминокислотной заменой описана Úsifo и соавторами [11]. Ранее не была описана мутация неуточненной клинической значимости p.Ala776Ser, расположенная в 16-м экзоне. Редкие миссенс-варианты данной локализации распространены в общей популяции, и большинство из них классифицируются как непатогенные, однако полностью исключить фенотип гиперхолестеринемии не представляется возможным [12]. Вероятно патогенная мутация p.Gly20Arg гена LDLR встречалась у двух пациентов. Патогенный вариант p.Glu208Lys описан у одного больного.

В гене $A P O B$ выявлены патогенные и вероятно патогенные мутации у двоих пациентов и еще у двоих обнаружены мутации неуточненной клинической значимости. Встречаемость всех определенных вариантов в общей популяции составляет менее 1\%.

В гене PCSK9 обнаружены ранее не описанные мутации неуточненной клинической значимости у двоих обследованных. Мутация p.Glu612Lys расположена в 11-м экзоне. Известно о связи мутаций в этом функциональном домене (цистеин-гистидин-обогащенный С-концевой домен) с СГХС. В частности, имеется модифицирующее влияние подобных генетических вариантов на пациентов, являющихся носителями мутаций в гене $L D L R$ (важно отметить, что обследованный нами больной также является носителем вероятно патогенной мутации p.Gly20Arg гена $L D L R$ ) [13]. Мутация с. ${ }^{*} 415 \mathrm{G}>\mathrm{A}$ расположена в $12-\mathrm{M}$ экзоне. Это нетранслируемый участок, который содержит элементы, отвечающие за экспрессию гена. Однако оценить функциональные последствия такого рода генетических изменений не представляется возможным.

У пациентов с клинически диагностированной СГХС встречались мутации и в других генах, участвующих в липидном обмене. Наиболее широко представлены изменения в гене $A P O E ;$ в нем были выявлены мутации у двоих пациентов. В частности, у одного из них имелась вероятно патогенная ранее не описанная мутация p.Arg160His с локализацией в 4-м экзоне. Этот участок отвечает за связывание липопротеина АРОЕ с ЛНПР, и уже известно об ассоциации вариантов данной локализации (с аналогичной аминокислотной заменой) с развитием аутосомно-доминантной семейной дисбеталипротеинемии [14]. Кроме того, у четверых пациентов имелся один и тот же полиморфизм гена APOE, отвечающий за образование атерогенной изоформы 44 аполипопротеина E (в одном из случаев одновременно с носительством мутации гена АРОВ неуточненной клинической значимости p.Ala4002Val).

У троих пациентов были обнаружены мутации в гене ABCA1, из них одна патогенная и две вероятно патогенные, обусловливающие сниженный уровень ЛВП. У двоих обследованных имелись мутации в гене ABCG8: патогенная и неуточненной клинической значимости, для которых описана связь с ситостеролемией [15]. Один больной имел вероятно патогенную мутацию в гене ABCG5, один - вероятно патогенную мутацию гена $L P L$, еще у одного была патогенная мутация в гене ANGPTL3 и у одного - мутация неуточненной клинической значимости в гене МTTP.

Таким образом, из 38 пациентов с клинически диагностированной СГХС у 24 (63,2\%) были выявлены генетические изменения, которые могли бы обусловить клинические проявления СГХС и раннюю манифестацию ИБС. Среди них 5 ранее описаны не были.

Результаты проведенного обследования представлены в таблице и на рис. 1 .

\section{ОБСУЖДЕНИЕ РЕЗУЛЬТАТОВ}

Частота встречаемости СГХС в общей популяции составляет 0,2-0,5\%, а среди пациентов с ОКС может достигать 8\%, в связи с чем ее адекватная диагностика именно в этой группе пациентов на сегодняшний день представляется актуальной проблемой, так как дает возможность своевременного назначения соответствующей липидснижающей терапии и проведения каскадного скрининга [16, 17]. Изучение распространенности различных мутаций, вызывающих развитие СГХС, в российской популяции находится только на начальном этапе [18]. Однако известно о крайней гетерогенности и отсутствии эффекта основателя, что было выявлено исследователями из Санкт-Петербурга [19]. В частности, в обследованной ими группе больных с СГХС (без ОКС) не было обнаружено носителей мутации гена $A P O B$. Еще в одном из исследований, проведенном также на северо-западе России, не выявлено мутаций в гене $A P O B$ [20]. В нашей выборке больных с «ранним» развитием OKC мутации в генах $L D L R$ и $A P O B$ представлены почти в одинаковой степени (четыре мутации гена $L D L R$, из которых три патогенных/вероятно патогенных, а одна встречается у двух пациентов; четыре мутации $A P O B$, из которых две патогенных/вероятно патогенных). Обращает также на себя внимание выявление в обследованной 
Таблица. Результаты секвенирования пациентов с «ранним» развитием ОКС и клинически диагностированной СГХС

\begin{tabular}{|c|c|c|c|c|c|c|c|c|c|}
\hline $\mathrm{N}$ & Пол & Возраст & $\begin{array}{l}\text { Наследс- } \\
\text { твенность }\end{array}$ & $\begin{array}{c}\text { ЛНП, } \\
\text { ммоль/л }\end{array}$ & $\begin{array}{l}\text { КГСЛК, } \\
\text { баллы }\end{array}$ & $\begin{array}{l}\text { СГХС Simon } \\
\text { Broome }\end{array}$ & Результат & $\begin{array}{c}\text { Патогенность } \\
\text { варианта }\end{array}$ & $\begin{array}{c}\text { Популяционная } \\
\text { частота }\end{array}$ \\
\hline 1 & ж & 48 & + & 9,6 & 11 & + & $\begin{array}{l}\text { Ген LDLRp.Val273_Cys313del; } \\
\text { c.817+303_940+943del }\end{array}$ & Патогенный & Не описана \\
\hline 2 & $M$ & 53 & - & 9,1 & 11 & + & Не обнаружено & - & - \\
\hline 3 & $M$ & 46 & + & 6,9 & 8 & + & $\begin{array}{l}\text { 1. Ген LDLR p.Gly20Arg; c. } 58 \mathrm{G}>\mathrm{A} \\
\text { 2. Ген PCSK9 p.Glu612Lys; } \\
\text { c. } 1834 \mathrm{G}>\mathrm{A}\end{array}$ & $\begin{array}{l}\text { 1. Вероятно патогенный } \\
\text { 2. Неуточненной } \\
\text { клинической значимости }\end{array}$ & $\begin{array}{l}\text { 1. }<1 \% \\
\text { 2. Не описан }\end{array}$ \\
\hline 4 & ж & 51 & + & 5,9 & 6 & + & $\begin{array}{l}\text { Полиморфизм гена } A P O E \\
\text { p.Cys130Arg; c.388T>C; } \\
\text { изоформа apoE4 }\end{array}$ & $\begin{array}{l}\text { Модифицирующий } \\
\text { фактор }\end{array}$ & $>1 \%$ \\
\hline 5 & $M$ & 46 & + & 5,6 & 6 & + & Не обнаружено & - & - \\
\hline 6 & $M$ & 49 & + & 5,1 & 6 & + & Не обнаружено & - & - \\
\hline 7 & ж & 57 & + & 5,7 & 6 & + & Ген LDLR p.Ala776Ser; c.2326G>T & $\begin{array}{l}\text { Неуточненной } \\
\text { клинической значимости }\end{array}$ & Не описан \\
\hline 8 & ж & 59 & + & 5,2 & 6 & + & Не обнаружено & - & - \\
\hline 9 & M & 52 & + & 6,2 & 6 & + & $\begin{array}{l}\text { 1. Полиморфизм гена } A P O E \\
\text { p.Сys130Arg; с.388T>C; } \\
\text { изоформа apoE4 } \\
\text { 2. Ген APOB p.Ala4002Val; c. } 12005 \mathrm{C}>\mathrm{T}\end{array}$ & $\begin{array}{l}\text { 1. Модифицирующий } \\
\text { фактор } \\
\text { 2. Неуточненной } \\
\text { клинической значимости }\end{array}$ & $\begin{array}{l}\text { 1. }>1 \% \\
\text { 2. }<1 \%\end{array}$ \\
\hline 10 & M & 55 & + & 6,2 & 6 & + & Ген PCSK9 с. ${ }^{\star} 415 \mathrm{G}>\mathrm{A}$ & $\begin{array}{c}\text { Неуточненной } \\
\text { клинической значимости }\end{array}$ & Не описан \\
\hline 11 & M & 54 & + & 6,5 & 6 & + & Ген ABCG8 p.Ala642Thr; c.G1924A & $\begin{array}{c}\text { Неуточненной } \\
\text { клинической значимости }\end{array}$ & $<1 \%$ \\
\hline 12 & M & 54 & + & 5,1 & 6 & + & Не обнаружено & - & - \\
\hline 13 & M & 36 & + & 5,6 & 6 & - & Ген APOE p.Arg180Cys; c.C538T & Вероятно патогенный & $<1 \%$ \\
\hline 14 & M & 34 & Неизвестна & 5,4 & 5 & + & $\begin{array}{l}\text { Полиморфизм гена } A P O E \\
\text { p.Cys130Arg; с.388T>C; } \\
\text { изоформа apoE4 }\end{array}$ & $\begin{array}{c}\text { Модифицирующий } \\
\text { фактор }\end{array}$ & $>1 \%$ \\
\hline 15 & M & 53 & Неизвестна & 5,2 & 5 & + & Не обнаружено & - & - \\
\hline 16 & ж & 56 & Неизвестна & 5,5 & 5 & - & Не обнаружено & - & - \\
\hline 17 & M & 44 & - & 5,8 & 5 & + & Ген $L D L R$ p.Gly20Arg; c.58G>A & Вероятно патогенный & $<1 \%$ \\
\hline 18 & M & 50 & - & 5,1 & 5 & + & Не обнаружено & - & - \\
\hline 19 & ж & 58 & - & 5,0 & 5 & - & Ген APOE p.Arg160His; c.479G>A & Вероятно патогенный & Не описана \\
\hline 20 & M & 55 & - & 6,3 & 5 & - & Не обнаружено & - & - \\
\hline 21 & M & 51 & + & 4,0 & 4 & + & $\begin{array}{l}\text { Полиморфизм гена } A P O E \\
\text { p.Cys130Arg; с.388T>C; } \\
\text { изоформа apoE4 }\end{array}$ & $\begin{array}{c}\text { Модифицирующий } \\
\text { фактор }\end{array}$ & $>1 \%$ \\
\hline 22 & ж & 57 & + & 4,0 & 4 & + & Ген $A B C A 1$ p.Pro85Leu; c.C254T & Вероятно патогенный & $<1 \%$ \\
\hline 23 & $\mathrm{M}$ & 43 & + & 4,9 & 4 & + & Ген LPL p.Thr379lle; c.C1136T & Вероятно патогенный & $<1 \%$ \\
\hline 24 & $M$ & 53 & + & 4,6 & 4 & + & Ген LDLR p.Glu208Lys; c.G622A & Патогенный & $<1 \%$ \\
\hline 25 & M & 47 & + & 5,4 & 6 & + & $\begin{array}{l}\text { Ген ANGPTL3 p.Asn } 147^{*} \text {; } \\
\text { c.431_434del }\end{array}$ & $\begin{array}{l}\text { Патогенный, семейная } \\
\text { гипобеталипопротеинемия }\end{array}$ & $<1 \%$ \\
\hline 26 & ж & 54 & + & 5,0 & 6 & + & Не обнаружено & - & - \\
\hline 27 & M & 39 & + & 5,3 & 6 & + & Ген ABCG5 p.Gly27Ala; c.G80C & $\begin{array}{l}\text { Вероятно патогенный, } \\
\text { ситостеролемия/ } \\
\text { гиперхолестеринемия }\end{array}$ & $<1 \%$ \\
\hline 28 & M & 46 & + & 6,5 & 6 & + & Ген MTTP p.Leu838Phe; c.G2514C & $\begin{array}{c}\text { Неуточненной } \\
\text { клинической значимости }\end{array}$ & $<1 \%$ \\
\hline 29 & $M$ & 45 & + & 5,0 & 6 & + & Не обнаружено & - & - \\
\hline 30 & M & 42 & + & 4,8 & 6 & - & Не обнаружено & - & - \\
\hline 31 & M & 51 & Неизвестна & 5,2 & 5 & + & Ген ABCG8 p.Leu572Pro; c.T1715C & $\begin{array}{c}\text { Патогенный, } \\
\text { ситостеролемия }\end{array}$ & $<1 \%$ \\
\hline 32 & ж & 47 & Неизвестна & 5,7 & 5 & - & Ген APOB p.Val4265Ala; с.T12794C & Вероятно патогенный & $<1 \%$ \\
\hline 33 & M & 48 & - & 4,9 & 5 & - & Не обнаружено & - & - \\
\hline 34 & ж & 59 & + & 4,9 & 4 & + & Ген ABCA1 p.Val399Ala; c.T1196C & $\begin{array}{c}\text { Патогенный, врожденный } \\
\text { дефицит ЛВП }\end{array}$ & $<1 \%$ \\
\hline 35 & M & 56 & + & 6,5 & 7 & - & Не обнаружено & - & - \\
\hline 36 & $\mathrm{M}$ & 64 & + & 5,0 & 6 & + & Ген $A P O B$ p.Ser3279Gly; c.A9835G & Вероятно патогенный & $<1 \%$ \\
\hline 37 & M & 57 & + & 5,8 & 6 & + & Ген АРОВ p.Asn2971Thr; с.A8912C & $\begin{array}{c}\text { Неуточненной } \\
\text { клинической значимости }\end{array}$ & $<1 \%$ \\
\hline 38 & ж & 64 & + & 5,6 & 5 & - & Ген $A B C A 1$ p.Arg496Trp; c.C1486T & $\begin{array}{c}\text { Вероятно патогенный, } \\
\text { врожденный дефицит ЛВП }\end{array}$ & $<1 \%$ \\
\hline
\end{tabular}




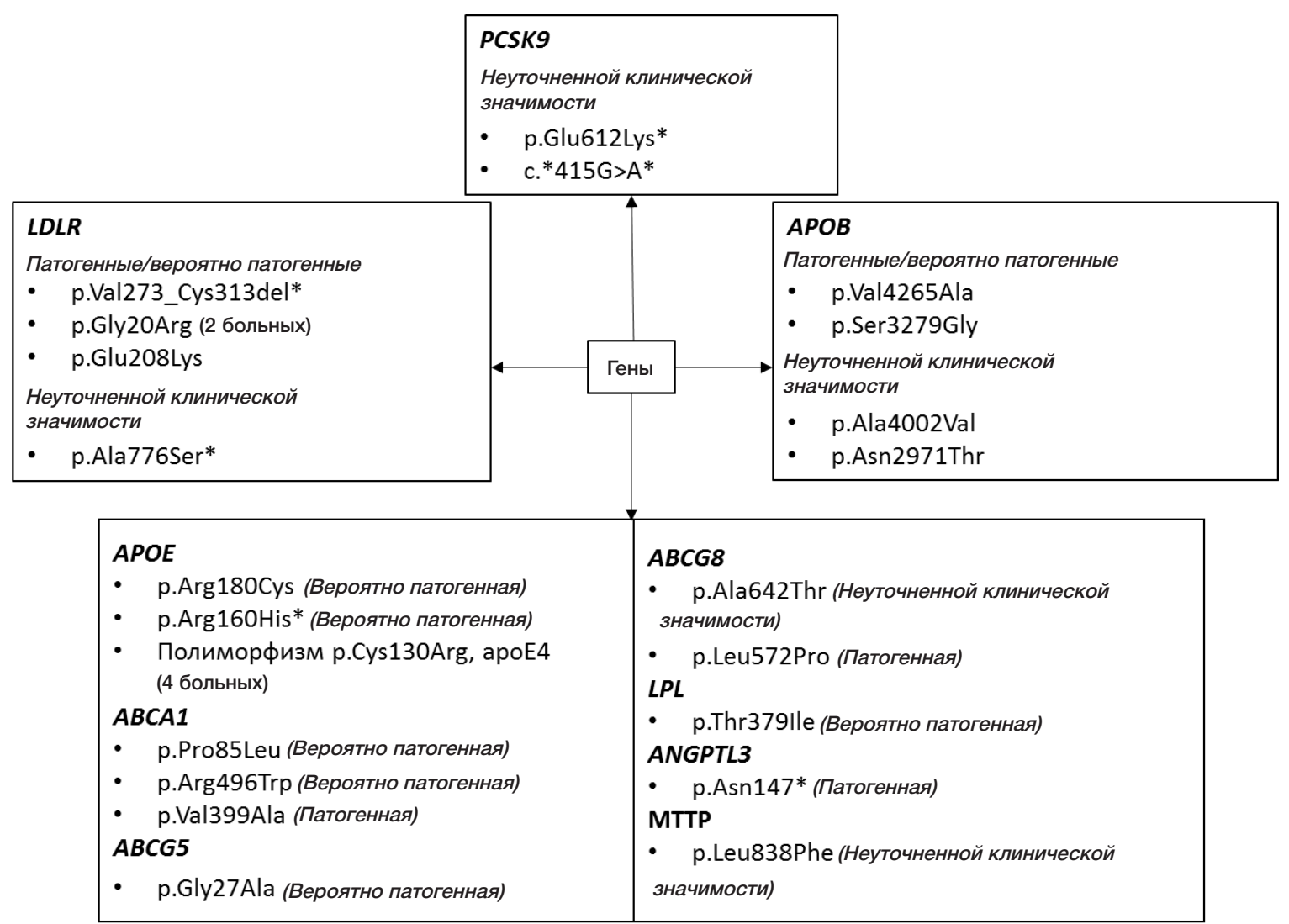

* Генетический вариант ранее не описан

Рис. 1. Генетические варианты, ассоциированные с клинически диагностированной СГХС у больных «ранним» ОКС

нами группе двух больных с фенотипом СГХС, которые являются носителями мутаций с неизвестной клинической значимостью в гене PCSK9. На сегодняшний день в России описание случаев мутаций PCSK9 пока единично [21]. Все известные патологические варианты гена PCSK9 являются чрезвычайно редкими и имеют аллельную частоту менее 0,1\% в общей популяции [22]. Распределение в общей популяции однонуклеотидных полиморфизмов, мало влияющих на обмен холестерина, такое, что у большинства наблюдается баланс между аллелями, повышающими и снижающими уровень ЛНП. У людей же, находящихся в крайних точках этого распределения, имеется наследственно обусловленное преобладание аллелей, повышающих уровень ЛНП, которые кумулятивно могут приводить к достижению значений ЛНП, характерных для гетерозиготной СГХС. Одним из самых характерных и изученных примеров такого полиморфизма является полиморфизм гена APOE $\varepsilon 2 / \varepsilon 3 / \mathrm{e} 4$, когда у носителей $\varepsilon 4$ риск ИБС в несколько раз выше [23, 24]. Известно об ассоциации носительства генотипа изоформ $\varepsilon 4$ полиморфного маркера p.Cys130Arg с развитием СГХС в популяции России [25], что подтверждается и нашими данными, где этот полиморфизм обнаружен у четверых больных. Важно также обратить внимание на широкую представленность редких мутаций в других генах липидного обмена (APOE, ABCA1, ABCG5, ABCG8, LPL, ANGPTL3, MTTP), что может указывать на возможно иную распространенность мутаций в популяции больных с «ранним» развитием ОКС и СГХС в России. Среди обследованных у пациентки № 1 (см. таблицу) ранее не описанная мутация p.Val273_Cys313del в гене LDLR носила определенно патогенный характер [11]. Был проведен каскадный скрининг у дочери пробанда (известно о повышении у нее уровня общего холестерина), не выявивший носительства патологического варианта гена. Итак, из 38 обследованных у 14 пациентов (36,8\%) выявленные генетические варианты носили патогенный и вероятно патогенный характер, а у 10 (26,3\%) имели неуточненную клиническую значимость. Данные о частоте встречаемости генетических вариантов, связанных с СГХС именно у больных с «ранним» ОКС, в литературе не описаны, однако, согласно исследованию ряда авторов, проводивших таргетное секвенирование у 104 пациентов с клинически диагностированной СГХС, были выявлены патологические варианты как в генах $L D L R, A P O B, P C S K 9$, так и в редких генах системы липидного обмена у $67 \%$ обследованных с определенной СГХС [26].

\section{ВЫВОДЫ}

Использование клинических критериев позволяет с помощью последующего таргетного секвенирования выявить среди пациентов с ОКС и СГХС носителей патологических генетических вариантов не только в «классических» генах, связанных с СГХС, но и в редких, которые, возможно, ассоциированы с фенотипическими проявлениями СГХС. 


\section{Литература}

1. Braenne I, Kleinecke M, Reiz B, Graf E, Strom T, Wieland T et al. Systematic analysis of variants related to familial hypercholesterolemia in families with premature myocardia infarction. Europ J Hum Genet. 2016; 24 (2): 191-7.

2. Sharifi M, Futema M, Nair D, Humphries SE. Genetic Architecture of Familial Hypercholesterolaemia. Current Cardiology Reports. 2017; 19 (5): 1-8.

3. Defesche JC, Gidding SS, Harada-Shiba M, Hegele RA, Santos RD, Wierzbicki AS. Familial hypercholesterolaemia. Nature Reviews Disease Primers. 2017; (3): 1-20.

4. Ouguerram K, Chetiveaux M, Zair Y, Costet P, Abifadel M, Varret $\mathrm{M}$ et al. Apolipoprotein B100 metabolism in autosomaldominant hypercholesterolemia related to mutations in PCSK9. Arteriosclerosis, Thrombosis, and Vascular Biology. 2004; 24 (8): 1448-53.

5. Sun H, Samarghandi A, Zhang N, Yao Z, Xiong M, Teng BB. Proprotein convertase subtilisin/kexin type 9 interacts with apolipoprotein B and prevents its intracellular degradation, irrespective of the low-density lipoprotein receptor. Arteriosclerosis, Thrombosis, and Vascular Biology. 2012; 32 (7): 1585-95.

6. Затейщиков Д. А., Волкова Э. Г., Гузь И. О., Евдокимова М. А., Асейчева О. Ю., Галявич А. С. и др. Лечение больных, перенесших острый коронарный синдром, по данным Российского многоцентрового проспективного наблюдательного исследования. Фарматека. 2009; (12): 109-13.

7. Catapano AL, Graham I, De Backer G, Wiklund O, Chapman MJ, Drexel $\mathrm{H}$ et al. 2016 ESC/EAS Guidelines for the Management of Dyslipidaemias. European Heart Journal. 2016; 37 (39): 29993058.

8. Wierzbicki AS, Humphries SE, Minhas R. Familial hypercholesterolaemia: summary of NICE guidance. British Medical Journal. 2008; (337): 1095.

9. Richards S, Aziz N, Bale S, Bick D, Das S, Gastier-Foster J et al. Standards and guidelines for the interpretation of sequence variants: a joint consensus recommendation of the American College of Medical Genetics and Genomics and the Association for Molecular Pathology. Genet Med. 2015; 17 (5): 405-424.

10. Аверкова А. О., Бражник В. А., Королева О. С., Зубова Е. А., Рогожина А. А., Хасанов Н. Р. и др. Особенности поражения коронарного русла и течения острого коронарного синдрома у молодых больных с семейной гиперлипидемией по данным наблюдательного проекта ОРАКУЛ ІІ. Комплексные проблемы сердечно-сосудистых заболеваний. Приложение. 2017; 6 (4): 9.

11. Usifo E, Leigh SEA, Whittall RA, Lench N, Taylor A, Yeats C et al. Low-Density Lipoprotein Receptor Gene Familial Hypercholesterolemia Variant Database: Update and Pathological Assessment. Annals of Human Genetics. 2012; 76 (5): 387-401.

12. Bertolini S, Pisciotta L, Rabacchi C, Cefalu AB, Noto D, Fasano T et al. Spectrum of mutations and phenotypic expression in patients with autosomal dominant hypercholesterolemia identified in Italy. Atherosclerosis. 2013; 227 (2): 342-8.

13. Pisciotta L, Priore Oliva C, Cefalu AB, Noto D, Bellocchio A, Fresa R et al. Additive effect of mutations in LDLR and PCSK9 genes on the phenotype of familial hypercholesterolemia. Atherosclerosis. 2006; 186 (2): 433-40.

14. Wintjens R, Bozon D, Belabbas K, MBou F, Girardet JP, Tounian PA et al. Global molecular analysis and APOE mutations in a cohort of autosomal dominant hypercholesterolemia patients in France. Journal of Lipid Research. 2016; 57 (3): 482-91.

\section{References}

1. Braenne I, Kleinecke M, Reiz B, Graf E, Strom T, Wieland T et al. Systematic analysis of variants related to familia hypercholesterolemia in families with premature myocardial infarction. Eur J Hum Genet. 2016; 24 (2): 191-7.

2. Sharifi M, Futema M, Nair D, Humphries SE. Genetic Architecture of Familial Hypercholesterolaemia. Current Cardiology Reports. 2017; 19(5): 1-8.
15. Lu K, Lee MH, Hazard S, Brooks-Wilson A, Hidaka H, Kojima H et al. Two genes that map to the STSL locus cause sitosterolemia: genomic structure and spectrum of mutations involving sterolin-1 and sterolin-2, encoded by ABCG5 and ABCG8, respectively. Am J Hum Genet. 2001; 69 (2): 278-90.

16. De Backer G, Besseling J, Chapman J, Hovingh GK, Kastelein JJP, Kotseva $K$ et al. Prevalence and management of familial hypercholesterolaemia in coronary patients: An analysis of EUROASPIRE IV, a study of the European Society of Cardiology. Atherosclerosis. 2015; 241 (1): 169-75.

17. Nordestgaard BG, Chapman MJ, Humphries SE, Ginsberg HN, Masana L, Descamps OS et al. Familial hypercholesterolaemia is underdiagnosed and undertreated in the general population: guidance for clinicians to prevent coronary heart disease: consensus statement of the European Atherosclerosis Society. European Heart Journal. 2013; 34 (45): 3478-90a.

18. Ежов М. В., Сергиенко И. В., Дупляков Д. В., Абашина О. Е., Качковский М. А., Шапошник И. И. и др. Результаты Российской научно-исследовательской программы по диагностике и лечению больных семейной гиперхолестеринемией. Высокая распространенность, низкая информированность, плохая приверженность. Атеросклероз и дислипидемии. 2017; (2): $5-15$.

19. Захарова Ф. М., Татищева Ю. А., Голубков В. И., Липовецкий Б. М., Константинов В. О., Денисенко А. Д. и др. Семейная гиперхолестеринемия в Санкт-Петербурге: разнообразие мутаций свидетельствует об отсутствии выраженного эффекта основателя. Генетика. 2007; 43 (9): 1255-62.

20. Корнева В. А., Богословская Т. Ю., Кузнецова Т. Ю., Мандельштам М. Ю., Васильев Б. В. Семейная гиперхолестеринемия, обсуловленная новой мутацией гена рецепторов липопротеинов низкой плотности. Клиническая медицина. 2014; (7): 49-53.

21. Шахтшнейдер Е. В., Макаренкова К. В., Астракова К. С., Иванощук Д. Е., Орлов П. С., Рагино Ю. И. и др. Таргетное секвенирование гена PCSK9 у пациентов семейной гиперхолестеринемией в России. Кардиология. 2017; (6): 46-51.

22. Kosenko T, Golder M, Leblond G, Weng W, Lagace TA. Low density lipoprotein binds to proprotein convertase subtilisin/kexin type-9 (PCSK9) in human plasma and inhibits PCSK9-mediated low density lipoprotein receptor degradation. The Journal of biological chemistry. 2013; 288 (12): 8279-88.

23. Bennet AM, Di Angelantonio E, Ye Z, Wensley F, Dahlin A, Ahlbom A et al. Association of apolipoprotein $E$ genotypes with lipid levels and coronary risk. Jama. 2007; 298 (11): 1300-11.

24. Luo JQ, Ren H, Banh HL, Liu MZ, Xu P, Fang PF et al. The Associations between Apolipoprotein E Gene Epsilon2/Epsilon3/ Epsilon4 Polymorphisms and the Risk of Coronary Artery Disease in Patients with Type 2 Diabetes Mellitus. Frontiers in physiology. 2017; (8): 1031.

25. Shakhtshneider EV, Ragino YI, Chernjavski AM, Kulikov IV, Ivanova MV, Voevoda MI. Apolipoprotein E gene polymorphism in men with coronary atherosclerosis in Siberia. Bulletin of experimental biology and medicine. 2011; 150 (3): 355-8.

26. Vandrovcova J, Thomas ER, Atanur SS, Norsworthy PJ, Neuwirth C, Tan $Y$ et al. The use of next-generation sequencing in clinical diagnosis of familial hypercholesterolemia. Genet Med. 2013; 15 (12): 948-57.
3. Defesche JC, Gidding SS, Harada-Shiba M, Hegele RA, Santos RD, Wierzbicki AS. Familial hypercholesterolaemia. Nature Reviews Disease Primers. 2017; (3): 1-20.

4. Ouguerram K, Chetiveaux M, Zair Y, Costet P, Abifadel M, Varret M et al. Apolipoprotein B100 metabolism in autosomal-dominant hypercholesterolemia related to mutations in PCSK9. Arteriosclerosis, Thrombosis, and Vascular Biology. 2004; 24 (8): 1448-53. 
5. Sun $\mathrm{H}$, Samarghandi $A$, Zhang $N$, Yao Z, Xiong $M$, Teng BB Proprotein convertase subtilisin/kexin type 9 interacts with apolipoprotein B and prevents its intracellular degradation, irrespective of the low-density lipoprotein receptor. Arteriosclerosis, Thrombosis, and Vascular Biology. 2012; 32 (7): 1585-95.

6. Zatejshhikov DA, Volkova JeG, Guz IO, Evdokimova MA, Asejcheva OJu, Galjavich AS i dr. Lechenie bol'nyh, perenesshih ostryj koronarnyj sindrom, po dannym Rossijskogo mnogocentrovogo prospektivnogo nabljudatel'nogo issledovanija. Farmateka. 2009; (12): 109-13.

7. Catapano AL, Graham I, De Backer G, Wiklund O, Chapman MJ, Drexel $\mathrm{H}$ et al. 2016 ESC/EAS Guidelines for the Management of Dyslipidaemias. Eur Heart Journal. 2016; 37 (39): 2999-3058.

8. Wierzbicki AS, Humphries SE, Minhas R. Familial hypercholesterolaemia: summary of NICE guidance. British Medical Journal. 2008; (337): 1095.

9. Richards S, Aziz N, Bale S, Bick D, Das S, Gastier-Foster J et al. Standards and guidelines for the interpretation of sequence variants: a joint consensus recommendation of the American College of Medical Genetics and Genomics and the Association for Molecular Pathology. Genet Med. 2015; 17 (5): 405-24.

10. Averkova AO, Brazhnik VA, Koroleva OS, Zubova EA, Rogozhina AA, Hasanov NR et al. Osobennosti porazheniya koronarnogo rusla i techeniya ostrogo koronarnogo sindroma u molodyh bol'nyh s semejnoj giperlipidemiej po dannym nablyudatel'nogo proekta ORAKUL II. Kompleksnye problemy serdechno-sosudistyh zabolevanii. Prilozhenie. 2017; 6 (4): 9.

11. Usifo E, Leigh SEA, Whittall RA, Lench N, Taylor A, Yeats C et al. Low-Density Lipoprotein Receptor Gene Familial Hypercholesterolemia Variant Database: Update and Pathological Assessment. Annals of Human Genetics. 2012; 76 (5): 387-401.

12. Bertolini S, Pisciotta L, Rabacchi C, Cefalu AB, Noto D, Fasano T et al. Spectrum of mutations and phenotypic expression in patients with autosomal dominant hypercholesterolemia identified in Italy. Atherosclerosis. 2013; 227 (2): 342-8.

13. Pisciotta L, Priore Oliva C, Cefalu AB, Noto D, Bellocchio A, Fresa R et al. Additive effect of mutations in LDLR and PCSK9 genes on the phenotype of familial hypercholesterolemia. Atherosclerosis. 2006; 186 (2): 433-40.

14. Wintjens R, Bozon D, Belabbas K, MBou F, Girardet JP, Tounian PA et al. Global molecular analysis and APOE mutations in a cohort of autosomal dominant hypercholesterolemia patients in France. Journal of lipid research. 2016; 57 (3): 482-91.

15. Lu K, Lee MH, Hazard S, Brooks-Wilson A, Hidaka H, Kojima H et al. Two genes that map to the STSL locus cause sitosterolemia: genomic structure and spectrum of mutations involving sterolin-1 and sterolin-2, encoded by ABCG5 and ABCG8, respectively. Am J Hum Genet. 2001; 69 (2): 278-90.

16. De Backer G, Besseling J, Chapman J, Hovingh GK, Kastelein JJP Kotseva $\mathrm{K}$ et al. Prevalence and management of familial hypercholesterolaemia in coronary patients: An analysis of EUROASPIRE IV, a study of the European Society of Cardiology. Atherosclerosis. 2015; 241 (1): 169-75.

17. Nordestgaard BG, Chapman MJ, Humphries SE, Ginsberg HN, Masana L, Descamps OS et al. Familial hypercholesterolaemia is underdiagnosed and undertreated in the general population: guidance for clinicians to prevent coronary heart disease: consensus statement of the European Atherosclerosis Society. European Heart Journal. 2013; 34 (45): 3478-90a.

18. Ezhov MV, Sergienko IV, Duplyakov DV, Abashina OE, Kachkovskiy MA, Shaposhnik II et al. Rezul'taty Rossijskoj nauchnoissledovatel'skoj programmy po diagnostike i lecheniyu bol'nyh semejnoj giperholesterinemiej. Vysokaya rasprostranennost', nizkaya informirovannost', plohaya priverzhennost'. Ateroskleroz i dislipidemii. 2017; (2): 5-15.

19. Zaharova FM, Tatishcheva YA, Golubkov VI, Lipoveckij BM, Konstantinov VO, Denisenko AD et al. Semejnaya giperholesterinemiya $v$ Sankt-Peterburge: raznoobrazie mutacif svidetel'stvuet ob otsutstvii vyrazhennogo ehffekta osnovatelya. Genetika. 2007; 43 (9): 1255-62.

20. Korneva VA, Bogoslovskaya TY, Kuznecova TY, Mandelshtam MY, Vasilev BV. Semejnaya giperholesterinemiya, obsulovlennaya novoj mutaciej gena receptorov lipoproteinov nizkoj plotnosti. Klinicheskaya medicina. 2014; (7): 49-53.

21. Shakhtshneider EV, Makarenkova KV, Astrakova KS, Ivanoshchuk DE, Orlov PS, Ragino Yl et al. Targetnoe sekvenirovanie gena PCSK9 u pacientov semejnoj giperholesterinemiej v Rossii. Kardiologiya. 2017; (6): 46-51.

22. Kosenko T, Golder M, Leblond G, Weng W, Lagace TA. Low density lipoprotein binds to proprotein convertase subtilisin/kexin type-9 (PCSK9) in human plasma and inhibits PCSK9-mediated low density lipoprotein receptor degradation. The Journal of biological chemistry. 2013; 288 (12): 8279-88.

23. Bennet AM, Di Angelantonio E, Ye Z, Wensley F, Dahlin A, Ahlbom A et al. Association of apolipoprotein $E$ genotypes with lipid levels and coronary risk. Jama. 2007; 298 (11): 1300-11.

24. Luo JQ, Ren H, Banh HL, Liu MZ, Xu P, Fang PF et al. The Associations between Apolipoprotein E Gene Epsilon2/Epsilon3/ Epsilon4 Polymorphisms and the Risk of Coronary Artery Disease in Patients with Type 2 Diabetes Mellitus. Frontiers in physiology. 2017; (8): 1031

25. Shakhtshneider EV, Ragino YI, Chernjavski AM, Kulikov IV, Ivanova MV, Voevoda MI. Apolipoprotein E gene polymorphism in men with coronary atherosclerosis in Siberia. Bulletin of experimental biology and medicine. 2011; 150 (3): 355-8.

26. Vandrovcova J, Thomas ER, Atanur SS, Norsworthy PJ, Neuwirth C, Tan $Y$ et al. The use of next-generation sequencing in clinical diagnosis of familial hypercholesterolemia. Genet Med. 2013; 15 (12): 948-57. 